\title{
Risikorechner zur Bestimmung des Frakturrisikos bei Osteoporose
}

Neuner-Jehle, S

Posted at the Zurich Open Repository and Archive, University of Zurich ZORA URL: https://doi.org/10.5167/uzh-93644

Journal Article

Published Version

Originally published at:

Neuner-Jehle, S (2013). Risikorechner zur Bestimmung des Frakturrisikos bei Osteoporose. PrimaryCare, $13(4): 69$. 


\title{
Risikorechner zur Bestimmung des Frakturrisikos bei Osteoporose
}

\author{
Ein Beitrag aus der Reihe «Die Werkzeugkiste des Familienarztes»
}

\begin{abstract}
Viele Frauen über 50 sind verunsichert, wenn sie in den Medien über die «Volkskrankheit Osteoporose» hören. Die Pharmaindustrie bewirbt ihre teuren Produkte im «Kampf gegen die Osteoporose» sehr aktiv. Wie gefährdet ist die Zielpopulation dieser Werbung wirklich? Braucht jede Frau über 50 eine Knochendichtemessung (DXA)? Risikoschätzer helfen hier weiter. Nehmen wir das Beispiel einer 62-jährigen Patientin mit Grössenabnahme/Keilwirbelbildung der Wirbelsäule, 165 cm / 59 kg, kalziumarme Ernährung, ohne weitere Osteoporose-Risikofaktoren.
\end{abstract}

\section{FRAX}

2007 extrahierte John Kanis aus Sheffield aus einer riesigen Kohorte von 290000 PatientInnen mit insgesamt 1,5 Millionen Patientenjahren, 18000 Frakturereignissen und 4314 Femurfrakturen die Daten, auf denen dieser Rechner beruht [1]. Der entscheidende Vorteil dabei ist, dass mit den wichtigsten Risikofaktoren für Osteoporose das Frakturrisiko errechnet werden kann, ohne die Knochendichte zu kennen (diese kann als zusätzlicher Parameter eingegeben werden). Neben biometrischen Fakten wie Alter, Geschlecht, Grösse und Gewicht sind anamnestische Angaben erforderlich: frühere Fraktur(en), Familienanamnese, Rauchen/ Alkohol und seltenere Osteoporosegründe (Steroidtherapie, Polyarthritis, sekundär verursachte Osteoporose). Nachteilig ist, dass schwierig quantifizierbare Mitursachen von osteoporotischen Frakturen wie z.B. die Sturzneigung nicht erfasst sind und dass theoretisch quantifizierbare Parameter (Anzahl Frakturen, Anzahl Zigaretten pro Tag) nicht differenziert sind. Das Ergebnis - die Frakturrate innert der nächsten 10 Jahre - ist unterteilt in irgendeine Lokalisation und in die Femurfraktur. Für unsere Beispielpatientin ergibt dies ein absolutes Risiko von 17\% bzw. 3,9\% (Femur) für das kommende Jahrzehnt.

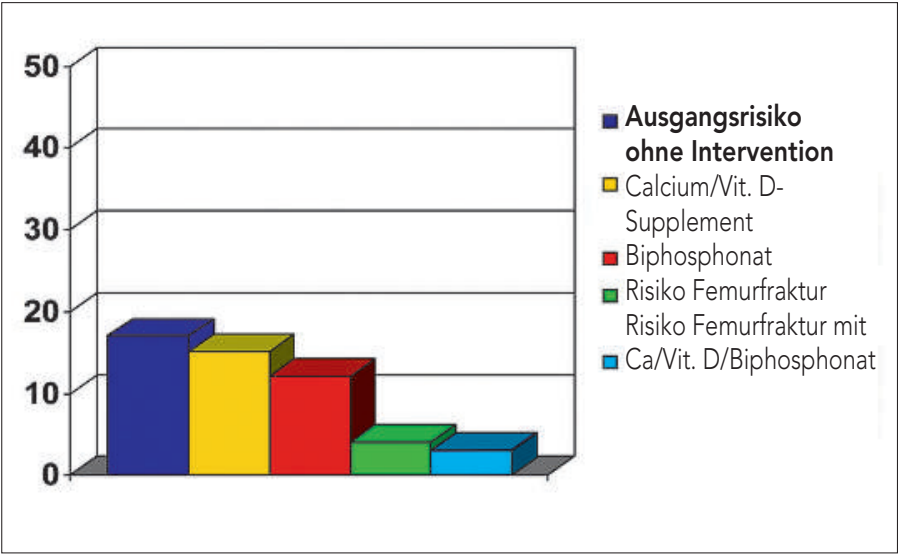

\section{Abbildung 1}

Visualisierung des Frakturrisikos. Mit FRAX errechnetes 10-Jahres-Frakturrisiko für unsere Beispielpatientin von 17\% für irgendeine Fraktur bzw. 3,9\% für eine Femurhalsfraktur (Ausgangsrisiken). Die Säulen zeigen, um wie viel das Risiko durch medikamentöse Interventionen gesenkt werden kann (Wirksamkeitsdaten bei [2]).
Interessant ist, dass die zusätzliche Angabe einer DXA im Osteopeniebereich die Frakturrate nicht dramatisch ändert,

zusätzliche ein oder zwei anamnestische Risiken hingegen einen deutlichen Unterschied ausmachen. Einmal mehr gilt: Anamnese ist relevanter als Medizintechnik.

\section{Osteo-Rheuma «Top»}

Auch die Schweizer Osteologen haben eine Anleitung entwickelt, welche Angaben zur Beurteilung einer möglichen Osteoporose nötig seien. Der umfangreiche Katalog, als Algorithmus dargestellt, gibt einen schönen Überblick über die Parameter, die dabei zu beachten sind. Aber: Braucht es wirklich all diese Abklärungen? Das vorgeschlagene Procedere des Tools für unsere Patientin: mehr Kalziumzufuhr, eine DXA, Labortests sowie eine Behandlung. Die Medikamente dafür werden am Ende des Pfades extensiv dargestellt. Einen Risikowert haben wir am Ende des Instruments nur in der Hand, wenn DXA-Werte verfügbar sind und eingegeben werden. Am Schluss lässt sich elegant ein Ausdruck der Angaben und Empfehlungen für die Patientin erstellen.

\section{Fazit}

Die Entwicklung von FRAX war ein Meilenstein, denn das Tool ermöglicht eine recht präzise Einschätzung ohne apparative Diagnostik. Aus meiner Sprechstunde ist es nicht mehr wegzudenken, und häufig können Niedrigrisiko-Patientinnen mit diesen Zahlen beruhigt werden, ohne dass eine DXA nötig wird. Ist der Entschluss für eine medikamentöse Therapie einmal gefallen, kommen wir allerdings wegen der SL-Limitationen und für die Verlaufsbeurteilung nicht um eine DXA herum.

Ohne viel Aufwand können die Risiken - analog zum kardiovaskulären Bereich - in einer Word-Datei visuell dargestellt oder einfach von Hand skizziert werden (Abb. 1). Damit versteht sie die Patientin besser und kann sich bewusster entscheiden.

Links zum Rechner bzw. zur Anleitung:

www.shef.ac.uk/FRAX

www.osteo-rheuma.ch/TOP

Literatur

1 Kanis JA, Johnell O, Oden A, Johansson H, McCloskey E. FRAXTM and the assessment of fracture probability in men and women from the UK. Osteoporos Int. 2008I;19(4):385-97.

2 Black DM, Cummings SR, Karpf DB, Cauley JA, Thompson DE, et al. Randomised trial of effect of alendronate on risk of fracture in women with existing vertebral fractures. Fracture Intervention Trial Research Group. Lancet. 1996;348(9041):1535-41. 